EPJ Web of Conferences 41, 04023 (2013)

DOI: $10.1051 /$ epjconf/20134104023

(C) Owned by the authors, published by EDP Sciences, 2013

\title{
Ultrafast low-energy dynamics of graphite studied by nonlinear multi-THz spectroscopy
}

\author{
C. Schmidt ${ }^{1}$, B. Mayer ${ }^{1}$, F. Junginger ${ }^{1}$, M. Rebholz ${ }^{1}$, A. Grupp ${ }^{1}$, D. Brida ${ }^{1,2}$, R. Huber ${ }^{1,3}$, \\ A. Leitenstorfer ${ }^{1}$, and A. Pashkin ${ }^{1}$ \\ ${ }^{1}$ Department of Physics and Center for Applied Photonics, University of Konstanz, \\ Universitätsstr. 10, 78464 Konstanz, Germany \\ ${ }^{2}$ IFN-CNR, Dipartimento di Fisica, Politecnico di Milano, Piazza Leonardo da Vinci 32, \\ 20133 Milano, Italy \\ ${ }^{3}$ Department of Physics, University of Regensburg, Universitätsstr. 31, 93053 Regensburg, Germany
}

\begin{abstract}
Ultraintense few-cycle $\mathrm{THz}$ pulses are employed to study the nonlinear response of graphite. A phase sensitive 2D spectroscopy setup is capable of detecting pump-induced transient changes as well as multi-wave mixing processes. The observed strong THz-pump THz-probe signals provide insight into ultrafast dynamics and the spectral response of the low-energy carriers. Here we report the observation of a pumpinduced transmission in graphite. The relaxation dynamics shows three distinct time scales, which are assigned to carrier thermalization, phonon emission and a slow cooling down back to equilibrium.
\end{abstract}

Graphite and graphene feature exceptional electronic and lattice properties that are important for applications in optoelectronics as well as for fundamental physics [1]. The electronic band structure of both materials shows a vanishing energy gap between valence and conduction bands which enables the study of massless charge carriers in the graphene case. $\mathrm{THz}$ pump / $\mathrm{THz}$ probe experiments performed with picosecond pulses from a free-electron laser have demonstrated an anomalous nonlinear response of carriers in the vicinity of the Dirac point of graphene [2].

Winnerl and coworks observed a pump-induced bleaching for a fluence of $\Phi \approx 1 \mu \mathrm{J} / \mathrm{cm}^{2}$ and photon energies exceeding twice the Fermi energy. Important insights into the relaxation dynamics in graphite have been obtained using near-infrared (NIR) excitation [3,4]. Two-dimensional femtosecond spectroscopy is expected to allow for a particularly direct observation of ultrafast dynamics and spectral correlations. At $\mathrm{THz}$ frequencies this technique promises access to the intriguing low-energy interband dynamics of graphite and graphene. In fact, lead-off experiments on $\mathrm{THz}$ multi-wave mixing have been proposed recently [5]. Latest advances in the multi-THz technology pave the way towards a systematic investigation of extreme $\mathrm{THz}$ nonlinearities utilizing

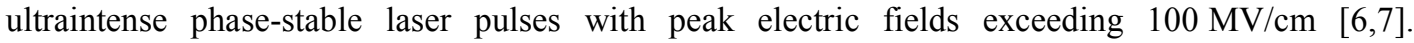
Furthermore, electro-optic detection warrants a direct observation of the time trace of the carrier field with absolute amplitude and phase resolution.

Here we employ our high-field multi-THz source to study the nonlinear response of a thin graphite flake using a two-dimensional spectroscopy setup. A highly oriented pyrolytic graphite (HOPG) crystal is exfoliated down to a thickness of approximately $130 \mathrm{~nm}$ (Figure 1(a)). The

This is an Open Access article distributed under the terms of the Creative Commons Attribution License 2.0, which permits unrestricted use, distribution, and reproduction in any medium, provided the original work is properly cited. 
sample is bonded to a $250-\mu \mathrm{m}$-thick diamond window via van der Waals forces. All experiments are performed at room temperature and the $\mathrm{THz}$ peak fields were chosen as high as $2.5 \mathrm{MV} / \mathrm{cm}$ per branch, corresponding to a fluence of $2 \mathrm{~mJ} / \mathrm{cm}^{2}$.

(a)

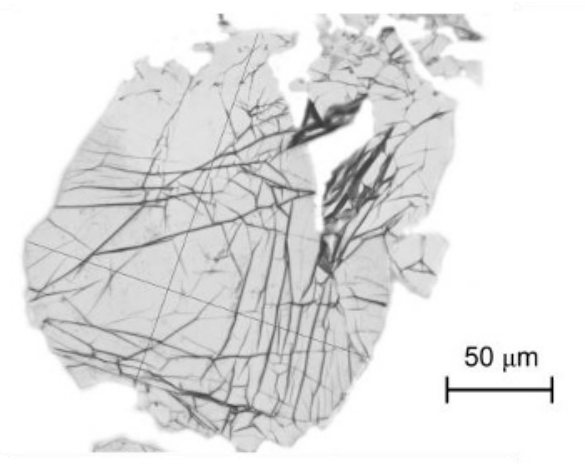

(b)

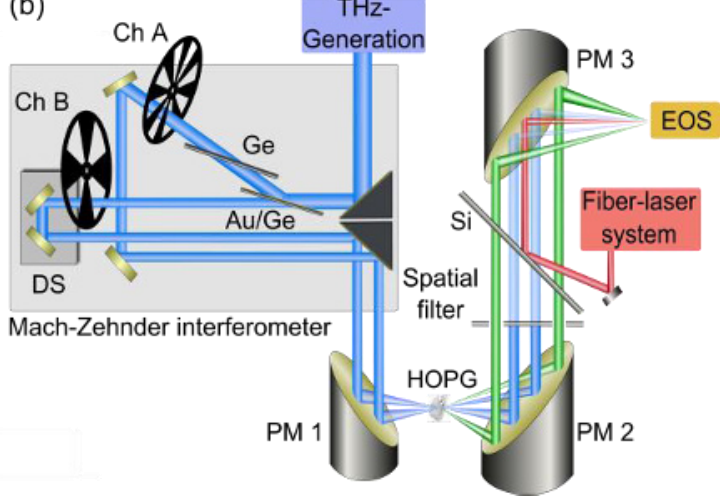

Fig. 1. (a) Optical microscope image of the 130-nm-thick graphite sample. (b) Two-dimensional multi-THz spectroscopy setup.

In our system, difference frequency mixing of two NIR pulse trains takes place in a GaSe crystal, thus providing intense $\mathrm{THz}$ transients centered at a frequency of $25 \mathrm{THz}$ with a bandwidth of $5 \mathrm{THz}$. A Ge beam splitter set at Brewster's angle and coated with a thin gold layer ( $\mathrm{Au} / \mathrm{Ge}$ ) provides two identical copies of the THz transient (Figure 1(b)). The reflected transient $E_{1}$ propagates through an additional Ge wafer to match the dispersion of the transmitted pulse $E_{2}$ which is delayed by a linear stage. Both beams are tightly focused onto the sample with an off-axis parabolic mirror. An electrooptic detection scheme is used to sample the emerging linear and nonlinear fields by a NIR gating pulse of a duration of $8 \mathrm{fs}$. The total transmitted field $E_{12}$ is retrieved as a function of the electrooptic sampling delay $t$ and the relative temporal offset $\tau$ between the THz pulses. The nonlinear signal $E_{\mathrm{NL}}$ is acquired by subtracting the individual transients $E_{1}$ and $E_{2}$ from the total response: $E_{\mathrm{NL}}=E_{12}-E_{1}-E_{2}$. We disentangle different nonlinear contributions, such as pump-probe and fourwave mixing signals, by two-dimensional spectral filtering of $E_{\mathrm{NL}}$ [5]. An appreciable pump-probe signal is clearly observed in our experiment (Figure 2(a)) whereas four-wave mixing and higher order multi-wave mixing signatures are too weak to be resolved, at present.

Figure 2(a) shows the transient change in $E_{1}$ induced by the field of $E_{2}$. A strong response in the temporal region where both transients overlap $(\tau=0 \mathrm{ps})$ is followed by a slower relaxation process. Owing to the field-resolved detection provided by electro-optic sampling we are able to extract the spectral profile of the pump-probe signal in the frequency range between 23 and $29 \mathrm{THz}$, covered by our broadband $\mathrm{THz}$ transients. Figure 2(b) depicts the amplitude spectrum of the normalized relative transmission change $\Delta \mathrm{T} / \mathrm{T}$ as a function of the pump-probe delay time. The dash-dotted line marks the change of sign. The ultrafast dynamics of $\Delta \mathrm{T} / \mathrm{T}$ at selected frequencies are plotted in Figure 2(c). Remarkably, at frequencies above $25 \mathrm{THz}$ the transmission change is negative in contrast to a positive differential transmission of multi-layer graphene in a similar frequency range that was reported recently [4]. This qualitative difference in the nonlinear $\mathrm{THz}$ response of graphite and graphene is likely related to the significant interlayer coupling in graphite which results in a modification of the band structure and additional interband optical transitions [8]. Theoretical modeling of the optical response of photodoped graphite is currently in progress.

The observed relaxation dynamics demonstrates at least three distinctly different time scales (Figure 2(c)): The fast initial relaxation within $\approx 200$ fs reflects the thermalization of the electron and hole distributions. The following decay of negative differential transmission on a 1-ps time scale is tentatively assigned to the cooling of the photogenerated charge carriers via electron optical-phonon scattering. Although the pump-photon energy is clearly below the optical-phonon emission threshold 
of $200 \mathrm{meV}$ the efficient carrier generation and strong electron-electron interaction lead to an extremely hot electron hole plasma with significant occupation of states with energy above 200 $\mathrm{meV}$. At longer delay times the pump-probe spectrum demonstrates a rather stable shape with a zerocrossing at a frequency of $24 \mathrm{THz}$ as shown in Figure 2(c) $(\tau=1.5 \mathrm{ps})$. At earlier times, the electron cooling dynamics results in the evolution of the pump-probe spectrum clearly revealed by the shift of the zero-crossing point depicted in Figure 2(b). For $\tau>1.5 \mathrm{ps}$, the intensity of the pump-probe signal decays slowly with a time constant distinctly larger than the measurement window of $3.5 \mathrm{ps}$. This relaxation reflects the cooling of charge carriers with a kinetic energy below the optical phonon emission threshold.

(a)

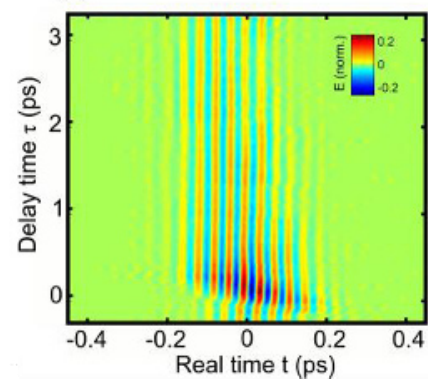

(b)

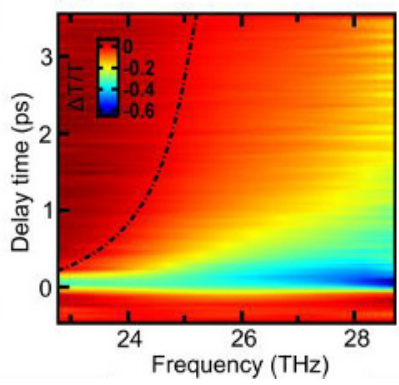

(c)

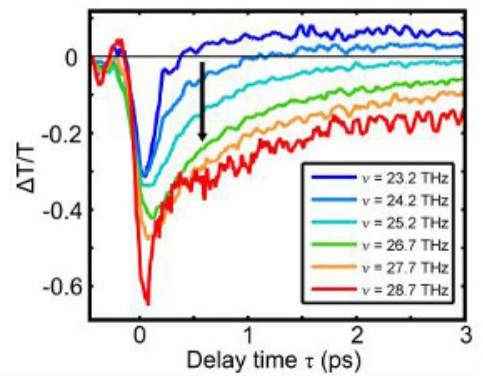

Fig. 2. (a) Pump-probe signal plotted as a function of the sampling time $t$ and the delay time $\tau$. (b) Relative transmission change $\Delta \mathrm{T} / \mathrm{T}$ of the probe transient plotted as a function of the frequency $v$ and the delay time $\tau$. The dashed-dotted line corresponds to $\Delta \mathrm{T} / \mathrm{T}=0$. (c) $\Delta \mathrm{T} / \mathrm{T}$ as a function of the delay time $\tau$ for different frequency components of the driving field. The arrow marks increasing frequencies.

Furthermore, we have performed measurements with $\mathrm{THz}$ transients centered at $30 \mathrm{THz}$. Surprisingly, the zero-crossing of the transient transmission spectra shifts towards higher frequencies by $5 \mathrm{THz}$, i.e. the same amount by which the THz pump pulse is changed (not shown). Thus, the $\mathrm{THz}$ pump-probe response in graphite is strongly dependent on the energy of the pump photons indicating a strong contribution of interband optical transitions compared to the response of the photogenerated plasma.

In conclusion, ultraintense few-cycle $\mathrm{THz}$ transients are used to study carrier dynamics in graphite. A strong pump-probe signal reveals three distinct relaxation processes of the $\mathrm{THz}$-induced transmission change. The transient transmission spectra demonstrate a characteristic zero-crossing which shifts on a picosecond time scale and depends on the central frequency of the pumping $\mathrm{THz}$ pulse. Our results demonstrate the high potential of the high-field multi-THz nonlinear spectroscopy for investigation of ultrafast carrier dynamics in graphite, graphene and related carbon-based materials.

1. A.K. Geim, K.S. Novoselov, Nature Mater. 6, 183 (2007)

2. S. Winnerl, M. Orlita, P. Plochocka, P. Kossacki, M. Potemski, T. Winzer, E. Malic, A. Knorr, M. Sprinkle, C. Berger, W.A. de Heer, H. Schneider, M. Helm, Phys. Rev. Lett. 107, 237401 (2011)

3. M. Breusing, C. Ropers, T. Elsaesser, Phys. Rev. Lett. 102, 086809 (2009)

4. T. Kampfrath, L. Perfetti, F. Schapper, C. Frischkorn, M. Wolf, Phys. Rev. Lett. 95, 187403 (2005)

5. W. Kuehn, K. Reimann, M. Woerner, T. Elsaesser, J. Chem. Phys. 130, 164503 (2009)

6. A. Sell, A. Leitenstorfer, R. Huber, Opt. Lett. 33, 2767 (2008)

7. F. Junginger, A. Sell, O. Schubert, B. Mayer, D. Brida, M. Marangoni, G. Cerullo, A. Leitenstorfer, R. Huber, Opt. Lett. 35, 2645 (2010)

8. A.B. Kuzmenko, E. van Heumen, F. Carbone, D. van der Marel, Phys. Rev. Lett. 100, 117401 (2008) 